\title{
Structure and function of FUS gene in prostate cancer
}

\author{
Ghanbarpanah E ${ }^{1}$, Kohanpour $\mathrm{MA}^{2}$, Hosseini-Beheshti $\mathrm{F}^{3}$, Yari L ${ }^{4}$, Keshvari $\mathrm{M}^{5}$ \\ Isfahan Cardiovascular Research Center, Cardiovascular Research Institute, Isfahan University \\ of Medical Sciences, Isfahan, Iran. Mahtabkeshvari87@yahoo.com
}

\begin{abstract}
BACKGROUND: FUS reduces the proliferator factors such as cyclin D1 and Cdk6, and increases Cdk and p27. Therefore, FUS prevents the growth of prostate cancer cells.

METHODS: This review tried to summarize data about FUS gene expression in correlation with the degree of prostate cancer. To find the relevant studies, the search in PubMed, Science Direct, and Scopus were performed. RESULTS: Increasing the expression of FUS decreases and increases the rate of apoptosis of prostate cancer cells, respectively. In fact, FUS reduces the proliferator factors such as: cyclin D1 and Cdk6, and increases Cdk (an anti-proliferation factor) and p27 (a proliferative inhibitory factor). Therefore, FUS prevents the growth of prostate cancer cells. An immuno-histochemical analysis showed that FUS gene expression had an inverse correlation with the degree of prostate cancer, which suggests that patients with higher levels of FUS are more likely to survive and less likely to have bone pain.

CONCLUSION: The key to FUS is the signaling of the androgen receptor and the progression of the cell cycle in prostate cancer. Based on these findings, we might be able to consider exogenous expression of FUS as a treatment for prostate cancer (Fig. 1, Ref. 32).

KEY WORDS: prostate cancer, FUS, androgen receptor, cyclin D1.
\end{abstract}

\section{Introduction}

The prostate gland is a small gland located below the bladder and covers the upper portion of the urethra. In developed countries, prostate cancer is the second most common cancer (after skin cancer) and the second leading cancer (after lung cancer) in men. Prostate malignancies were shown in 1 out of 6 people.

Epidemiological studies (epidemiology) showed that hereditary factors contribute to this disease in $10 \%$ of the cases $(1,2)$. The highest occurrence of prostate cancer was found in African population and the lowest numbers were found in the Asian population. Several studies were conducted on family history of prostate cancer. The main reason for this study is to investigate the involved genes. Prostate cancer is a disease, in which malignant cells pycnocline from prostate tissues erratically and increasingly proliferate and lead to an increase in prostate gland size (3).

It is estimated that more than 300,000 new cases are discovered every year, of which 41,000 definitely lead to death (3). Due to the high incidence of this disease in each community, special

${ }^{1}$ Department of Biology, Arsanjan Branch, Islamic Azad University, Arsanjan, Iran, ${ }^{2}$ Department of Physical Education, Lamerd Branch, Islamic Azad University, Lamerd, Iran, ${ }^{3}$ Medical Genetics Center of Genome, Isfahan University of medical Sciences, Isfahan, Iran, ${ }^{4}$ NIGEB National Institutes of Genetic Engineering and Biotechnology, and ${ }^{5}$ Isfahan Cardiovascular Research Center, Cardiovascular Research Institute, Isfahan University of Medical Sciences, Isfahan, Iran

Address for correspondence: Keshvari M, Isfahan Cardiovascular Research Center, Cardiovascular Research Institute, Isfahan University of Medical Sciences, Isfahan, Iran. attention to timely diagnosis and also effective treatment seems necessary.Prostate cancer is almost invariably dependent on the androgen receptor (AR) pathway, which,when activated, stimulates cell proliferation. Androgen receptor (AR) is required for the survival and growth of prostate cancer cells.

Prostate cancer is almost invariably related to the Androgen Receptor (AR) pathway, androgen receptor is a member of the receptor family of transcription factors. As soon as it is bonded to the androgen, the transcript of the androgen receptor regulates the target gene expression (4).

This receptor is activated when cell proliferation is stimulated. Several factors are involved in the progression of the cell cycle, which is regulated in response to androgens, for example, cyclin D1 increase (7-5). The endogenous amino zone (NTD) of the androgen receptor is essential for the activity of both the ligand and the unrelated receptor ligand (8). However, none of these cases of prostate cancer are treated with LHRH. Although the hormone blocks the production of androgens or anti-androgens, binds to AR and keeps it in an inactive state, this treatment is initially successful, later the treatment is failing and the tumor progresses. Most of the evidence illustrates that the receptor still grows in these conditions (9).

Androgen receptor (AR) is associated with survival and growth of prostate cancer cells. At first, it seemed that progressing prostate cancer could be treated with eradication of androgens.

Unfortunately, this treatment eventually fails, and the disease leads to death, which is called a castration resistant prostate cancer (CRPC). However, ongoing follow ups in the development of drugs can be useful for improving the understanding of the 
protein-protein interactions, which are involved in AR. In this regard, considering the genes involved in prostate cancer and interactions with the androgen receptor can lead to new and effective therapies in the future.

One of the new genes which are mentioned in this regard, is FUS RNA binding protein. This gene, characterized by FUS, is a kind of protein encoding gene. Also known as TLS, ALS6, ETM4, FUS1, POMP75 and HNRNPP2 (11). The gene encodes a multifunctional protein consisting of non-homogeneous nuclear ribonucleolase complex (hnRNP).

The hnRNP complex is involved in interacting with the premRNA and sending out of the mRNA product to the cytoplasm. This protein belongs to the FET family of RNA-binding proteins that play a role in cellular processes including the gene expression, genome protection, and the mRNA/ microRNA process [10].

\section{Methods for review}

An extensive search was performed in PubMed, Science Direct, Scopus and Google scholar to identify clinical and animal studies on the Structure and Function of FUS gene in prostate cancer published from inception up January 15, 2018. Search terms were ("prostate cancer" AND "FUS" AND "androgen receptor" AND "cyclin D1"). The search was performed in titles and abstracts that were restricted to articles published in English and sometimes French languages. All titles, abstracts, and full texts of potentially relevant studies were assessed for eligibility.

In this study, we mention the structure and function of the FUS, then review the related article, while explaining the various studies that have been done in the past few years, study the association of this gene with prostate cancer and the mechanism involved in this field. At the end, we give a final conclusion.

\section{Structure and function of the gene}

This gene (FUS) has 16 introns and 15 exons with16p11.2 chromosomal position, which is presented in Figure 1 (10).

Mutation in the FUS gene causes Amyotrophic Lateral Sclerosis (ALS) in $2009(11,12)$. In ALS disease, motor neurons have problems and lead to obstacle in muscle and movement control, at the end patient is unable to move (13). Approximately, about 40 mutations have been reported, most of which were present in the second richest $\mathrm{G}$ and $\mathrm{C}$-terminal NLS-containing region. The P525L mutation in the FUS is associated with the primary attack in ALS, in contrast, the subsequent attack of ALS is due to the R521C mutation in the FUS gene. Although the mutation in the FUS is responsible for only a small percentage of ALS (4.3\%), the FUS protein is a common cytoplasmic component in an ALS

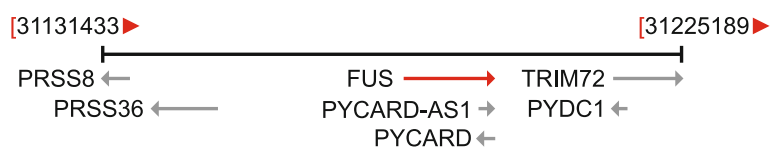

Fig. 1. FUS chromosome position. that is unrelated to SOD1 (most ALS cases are due to mutations in SOD1). The common FUS mutations that cause ALS include R521C (11, 12, 14), R521H (11, 12), H517Q (15), R521S (16), R514S (11), and R521L (15). . All of the FUS mutations occur in Axon 15 (16).

Also, the mutation S462F has recently been reported and recently known one polymorphism called Q210H, all associated with ALS. It has been suggested that ALS is associated with single nucleotide polymorphisms (SNPs) in various genes such as FUS (17).

The FUS encodes a multi-functional protein that requires the pre-mRNA interconnections (18), has a stable chromosome (19), expands the cell (20), and transcribes $(22,21)$.

The gene is member of a protein cell group called FET / TET. This family of proteins includes Fused in Sarcoma (FUS) / Translocated in LipoSarcoma (TLS), EWig, Sarcoma Protein (EWS), TATA binding protein-Associated Factor, and TAF15. FET proteins showed various functions including transcriptional combinations, binding, cell propagation, and DNA modification.

FET proteins have similar transcriptional active domains (TADs) at the end of the second amino acid (NTD) and have a main form of detected RNA (RNA recognition motif or RRM) and repeat the RGG tripeptide at the end of their carboxyl $(23,24)$. It has been shown, that FUS have interaction with DNA bandingdomains (DBDs) of retinoid X receptors, estrogen, thyroid, and glucocorticoid receptors (25).

FUS bonding to other DBDs of hormone receptors does not interfere with DNA bonding activity, although the role of the FUS in its transcriptional activity has not been clear (25).It has also been shown that FUS has a strong active transfer site that is functionally active in the prostate cancer cells (4).

\section{Gene and cancer correlation - mechanism and review of re- search}

As stated, the AR target is involved in the growth of prostate cancer cells therefore is valuable in recognition of new therapies. On the other hand, the FUS reduces a target protein of AR in response to androgen. Increasing the expression of FUS significantly delayed growth of androgen-caused prostate cancer cells both in in-vivo and in-vitro.

Regulation of expression of several factors, which are involved in the cell cycle (such as cyclin D1), FUS effect on them, prevents the entry into the G1 phase and the activation of apoptosis. Thus, the FUS illustrate tumor suppression characteristics.

Brooke et al (26) performed a proteomic imaging of $\mathrm{LNCaP}$ of the prostate cancer cell following stimulation with androgen and results showed a decrease in FUS expression at the level of RNA and protein.

Perrotti et al (27) showed that FUS was regulated at the protein level by c-jun, and Velasco et al (28) reported that c-jun was regulated by androgen. In support of these findings, Brooke et al (26) observed an increase in c-jun protein after eight hours of androgen injection. Whereas FUS decreased with increasing c-jun, scientists assumed that androgen might reduce FUS by increasing c-jun. However, c-jun suppression did not have an effect on 


\section{$660-663$}

the decrease in androgen receptors associated with FUS. Scientists concluded that reducing FUS in response to androgens was done at the transcriptional level (26). As FUS levels decreased by increasing androgen receptors, scientists assumed, FUS might be a suppressor of prostate cancer. In LNCaP cells, it was observed that FUS expression significantly prevented cell growth and this is because the FUS expression causes inhibition of the G1 phase and apoptosis increase (26).

It has been shown that $\mathrm{AR}$ played an important role in regulating regulatory factors in development of the cell cycle, especially in development of G1 / S, since androgen depletion can inhibit G1 (29). According to these findings, Brooke et al (26) reported that in $\mathrm{LNCaP}$ cells followed the elimination of androgens, prevented from entering the G1 phase, while the addition of androgen led to an increase in number of progressive cells to $S$ and G2 / M phases. In any case, the increased expression of FUS blocked the effect of androgen and prevented the G1 phase and also increased the number of the G1 subtypes.

Increasing activity of caspases and division of PARP confirmed that this G1 subtypes contained apoptotic cells, consequently, FUS increased apoptosis in prostate cancer cells (26).

The analysis of the cell cycle regulators showed that manipulation of FUS levels affected the expression of several important factors, especially cyclin D1, CDK 6 and P 27 in the G1 / S stage. It has been shown that cyclin D1 and P27 were targets for androgen, which caused an increase in cyclin D1 and a decrease in P 27 and also the prolongation of G1 stage (29-31). It has also been observed that the expression of exogenous FUS resulted in a decrease in expression of cyclin D1 and increase in the expression of P 27, which suggests that FUS inhibits G1 and thus inhibits androgenetic proliferation, which is partly through mediation of cyclin D1 and P 27 factors.

FUS directly uses the CCND 1 regulatory area encoding the cyclin D1 by stranded noncoding RNA (ncRNA) that is transcribed from different locations in the / 5 upstream region.

This effect results in interference with the complex transcriptional complexion, and hence the expression of cyclin D1 decreases (27). The cyclin D1 regulation has been observed in response to increasing or decreasing FUS at the level of RNA.

Brooke et al (26) showed that FUS levels were regulated by androgens, and Canduson et al (32) showed that reduction of their cyclin D1 was an androgen receptor suppressor. Therefore, there is a complex interaction between FUS, androgen receptor and cyclin D1, which should be further investigated in the future. However, it has been shown that the manipulation of FUS levels affected multiple levels of regulatory protein in the cell cycle, and it has been shown that FUS might be a vital link between androgen signaling and cell cycle progression.

Information from both the laboratory and the human body has shown that FUS has anti-tumor features (26).

FUS expression in the prostate tumor samples is correlated with the degree of prostate cancer, and patient information analysis showed that patient with higher FUS levels had the chance of more survival and lower bone pain (a major cause of illness in Prostate cancer patients) (26).
Therefore, the lack of FUS expression might be important in the progression of the disease. Although increased levels of FUS have reduced tumor growth, with a deletion of FUS inducing expression, tumor growth has increased again, while the FUS repression has reduced tumor volume by one-half a week (26).

\section{Conclusion}

Androgen signaling reduces FUS and subsequently increases the growth of prostate cancer cells as the result of important regulatory factors in the progression of the cell cycle.

In the same vein, FUS expression declines in the advanced stage of prostate cancer, therefore, loss of FUS may increase androgen signaling and subsequently increase the growth of prostate cancer cells. However, increased expression of FUS in the human body reduces tumor growth and prevents the G1 stage and increases apoptosis in prostate cancer cells.

Perhaps FUS induces this effect through the reductive effect on cyclin D1 and an increasing effect on P27. Therefore, FUS manipulation can be considered as a treatment for prostate cancer.

\section{References}

1. Carter H, Partin A. Diagnosis and staging of prostate cancer. Campbell's Urology. Sydney: Elsevier Science Publishers, 2002.

2. Jemal A, Murray T, Ward E, Samuels A, Tiwari RC, Ghafoor A et al. Cancer Statistics: American Cancer Society. CA Cancer J Clin 2005; 55: $10-30$.

3. Ramzi C, Kumar V, Collins T. Disease of Immunity. In: Robbins Pathologic Basis of Disease. Philadelphia: WB Saunders, 1999, 188-259.

4. Haile S, Lal A, Myung J, Sadar M. FUS/TLS Is a Co-Activator of Androgen Receptor in Prostate Cancer Cells. PLoS ONE 2011; 6: 1-9.

5. Cifuentes E, Croxen R, Menon M, Barrack E, Reddy G. Synchronized prostate cancer cells for studying androgen regulated events in cell cycle progression from G1 into S phase. J Cell Physiol 2003; 195: 337-345.

6. Knudsen K, Arden K, Cavenee W, Multiple G. Regulatory elements control the androgen-dependent proliferation of prostatic carcinoma cells. J Biol Chem 1998; 273: 20213-20222.

7. Chen Y, Robles A, Martinez L, Liu F, Gimenez-Conti I, Conti CJ. Expression of $\mathrm{G}_{1}$ cyclins, cyclin-dependent kinases, and cyclindependent kinase inhibitors in androgen-induced prostate proliferation in castrated rats. Cell Growth Differ 1996; 7: 1571-1578.

8. Wang G, Sadar M. Amino-terminus domain of the androgen receptor as a molecular target to prevent the hormonal progression of prostate cancer. J Cell Biochem 2006; 98: 36-53.

9. Feldman BJ, Feldman D. The development of androgen-independent prostate cancer. Nat Rev Cancer 2001; 1: 34-45.

10. www.ncbi.nIm.nih.gov/gene/2521.

11. Kwiatkowski T, Bosco D, Leclerc A, Tamrazian E, Vanderburg C, Russ C et al. Mutations in the FUS/TLS gene on chromosome 16 cause familial amyotrophic lateral sclerosis. Science 2009; 323: 1205-1208.

12. Vance C, Rogelj B, Hortobagyi T, De Vos K, Nishimura A, Sreedharan $\mathbf{J}$ et al. Mutations in FUS, an RNA processing protein, cause familial amyotrophic lateral sclerosis type 6. Science 2009; 323: 1208-1211. 
13. Loureiro J, Wilson K, De Mesquita J. In Silico Analysis and Structural Prediction of FUS Protein Polymorphisms in Amyotrophic Lateral Sclerosis Type 6. XLIII Annual Meeting of SBBq Foz do Iguaçu, PR, Brazil, May 17th to 20th, 2014.

14. Huang C, Zhou H, Tong J, Chen H, Liu Y, Wang D et al. FUS transgenic rats develop the phenotypes of amyotrophic lateral sclerosis and frontotemporal lobar degeneration. PLoS Genet 2011; 7: pp e1002011.

15. Yoshiaki F. Protein Aggregates in Pathological Inclusions of Amyotrophic Lateral Sclerosis, Amyotrophic Lateral Sclerosis, Prof. Martin Maurer (Ed.), 2012. Available from: www.intechopen.com/books/amyotrophic-lateral sclerosis/protein-aggregates-in-pathological-inclusionsofamyotrophic-lateral-sclerosis.

16. Millecamps S, Salachas F, Cazeneuve C, Gordon P, Bernard B et al. SOD1, ANG, VAPB, TARDBP, and FUS mutations in familial amyotrophic lateral sclerosis: genotype-phenotype correlations. J Med Genet 2010; 47: 554-560.

17. Fumoto K, Groen E, Vandenberg L, Pasterkamp J. Identification of FUS Interacting Proteins in Neuronal Cells. Amyotrophic Lateral Sclerosis 2010; 11: 84-95.

18. Meissner M, Lopato S, Gotzmann J, Sauermann G, Barta A. Protooncoprotein TLS/FUS is associated to the nuclear matrix and complexed with splicing factors PTB, SRm160, and SR proteins. Exp Cell Res 2003; 283: 184-195.

19. Hicks G, Singh N, Nashabi A, Mai S, Bozek G, Klewes L et al. FUS deficiency in mice results in defective B-lymphocyte development and activation, high levels of chromosomal instability and perinatal death. Nat Genet 2000; 24: 175-179.

20. Andersson M, Stahlberg A, Arvidsson Y, Olofsson A, Semb H, Stenman $\mathbf{G}$ et al. The multifunctional FUS, EWS and TAF15 protooncoproteins show cell type-specific expression patterns and involvement in cell spreading and stress response. BMC Cell Biol 2008; 9: 37.

21. Wang X, Arai S, Song X, Reichart D, Du K, Pascual G et al. Induced ncRNAs allosterically modify RNA-binding proteins in cis to inhibit transcription. Nature 2008; 454: 126-130.

22. Tan A, Manley J. TLS inhibits RNA polymerase III transcription. Mol Cell Biol 2010; 30: 186-196.
23. Law W, Cann K, Hicks G. TLS, EWS and TAF15: a model for transcriptional integration of gene expression. Brief Funct Genomic Proteomic 2006; 5: 8-14.

24. Tan A, Manley J. The TET family of proteins: functions and roles in disease. J Mol Cell Biol 2009; 1: 82-92.

25. Powers C, Mathur M, Raaka BM, Ron D, Samuels H. TLS (translocated-in-liposarcoma) is a high-affinity interactor for steroid, thyroid hormone, and retinoid receptors. Mol Endocrinol 1998; 12: 4-18.

26. Brooke G, Culley R, Dart D, Mann D, Gaughan L, McCracken S et al. FUS/TLS Is a Novel Mediator of Androgen-Dependent Cell-Cycle Progression and Prostate Cancer Growth. Cancer Res 2011; 71: 913-924.

27. Perrotti D, Iervolino A, Cesi V, Cirinná M, Lombardini S, Grassilli E et al. BCR-ABL prevents c-jun-mediated and proteasome-dependent FUS (TLS) proteolysis through a protein kinase CbetaII-dependent pathway. Mol Cell Biol 2000; 20: 6159-6169.

28. Velasco A, Gillis K, Li Y, Brown E, Sadler T, Achilleos M et al. Identification and validation of novel androgen-regulated genes in prostate cancer. Endocrinology 2004; 145: 3913-3924.

29. Knudsen K, Fribourg A, Petre C, Wetherill Y. Androgen ediated Regulation of the G1-S Transition in Prostate Cancer. In: Burnstein KL, editor. Steroid Hormones and Cell Cycle Regulation.Norwell, MA: Kluwer Academic Publishers, 2002, 91-110.

30. Lanzino M, Sisci D, Morelli C, Garofalo C, Catalano S, Casaburi I et al. Inhibition of cyclin D1 expression by androgen receptor in breast cancer cells-identification of a novel androgen response element. Nucleic Acids Res 2010; 38: 5351-5365.

31. Xu Y, Chen S, Ross K, Balk S. Androgens induce prostate cancer cell proliferation through mammalian target of rapamycin activation and post-transcriptional increases in cyclin D proteins. Cancer Res 2006; 66: 7783-7792.

32. Knudsen K, Cavenee W, Arden K. D-type cyclins complex with the androgen receptor and inhibit its transcriptional transactivation ability. Cancer Res 1999; 59: 2297-2301.

Received May 20, 2018. Accepted June 11, 2018. 